EISSN: 2706-7955 ISSN: 2077-4605

DOI: 10.36632/mejar/2021.10.1.6

Journal homepage: www.curresweb.com

Pages: 92-98

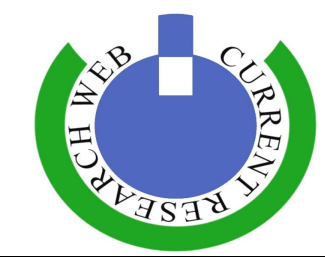

\title{
Efficiency of Nitrogen and Potassium Use and Its Relationship to the Addition of NP K Fertigation of Seqae Date Palm
}

${ }^{1}$ Abd Elhamid N.S. and ${ }^{2}$ Hamdy A. Mahdy

${ }^{1}$ Forestry and Timber Trees Res. Dept., Hort. Res. Inst., Agric. Res. Center, Egypt.

${ }^{2}$ Tropical Fruits Res. Dept., Hort. Res. Inst. Agric. Res. Center, Giza Egypt.

Received: 08 December 2020 Accepted: 05 January $2021 \quad$ Published: 25 January 2021

\begin{abstract}
The experimental was carried out on Seqae date palm (semi- dry cv.) Produced from tissue culture during three successive seasons $(2018,2019,2020)$ grown at El- Dakhala Oasis, Al- Wady Al - Jadid. The palms were received different rates of N, P, K fertigation. The annual rates per palm were 500,750 or $1000 \mathrm{~g}$ actual $\mathrm{N}$ in the form of $\mathrm{NH}_{4} \mathrm{NH}_{3}$, P fertigation rates $\left(400 \& 800 \mathrm{~g} \mathrm{P}_{2} \mathrm{O}_{5} /\right.$ palm/ year) as $\mathrm{H} 3 \mathrm{PO} 4$ and $\mathrm{K}$ - fertigation rates $\left(600 \& 1200 \mathrm{~g} \mathrm{~K}_{2} \mathrm{O} / \mathrm{palm} /\right.$ year). Results could be summarized as follows. Nitrogen use efficiency (NUE) values (the amount of date palm fruits in Kgs. that could be produced from one $\mathrm{Kg}$ of nitrogen) were effected with the NPK fertigation. Treatment $4(500 \mathrm{~g} \mathrm{~N}+800 \mathrm{~g}$ $\mathrm{P}_{2} \mathrm{O}_{5}+1200 \mathrm{~g} \mathrm{~K}_{2} \mathrm{O} / \mathrm{palm} /$ year) gave the highest values $198,206,333 \mathrm{kgs}$. Fruits Seqae date palm/ one $\mathrm{kg}$. of nitrogen during three seasons. Treatment $12\left(1000 \mathrm{~g} \mathrm{~N}+800 \mathrm{~g} \mathrm{P}_{2} \mathrm{O}_{5}+1200 \mathrm{~g} \mathrm{~K}_{2} \mathrm{O} / \mathrm{palm} /\right.$ year$)$ produced $(118,124,132 \mathrm{~kg}$. fruits/ one $\mathrm{Kg}$ of nitrogen.) Potassium use efficiency (KUE) values (the amount of date palm fruits in $\mathrm{kgs}$. that could be produced from one $\mathrm{Kg}$. of potassium) were effected with the NPK fertigation. The uppermost N, P, K level gave KUE $(98.3,103.3,110 \mathrm{~kg}$ fruit/ one $\mathrm{Kg}$ of potassium) during three tested seasons. The application of the large amount of NPK increased the mineral content of leaves of Seqae date palm. Increasing $\mathrm{N}$ rates raised leaf $\mathrm{N}$ and Fe contents and decreased leaf $\mathrm{P}, \mathrm{K}, \mathrm{Ca}, \mathrm{Mg}$ and $\mathrm{Zn}$ content but had no significant effect on leaf Mn content. Leaf N, P, $\mathrm{K}, \mathrm{Ca}$ and $\mathrm{Mg}$ contents increased with increasing rate of $\mathrm{P}$ fertigation, but unaffected on leaf $\mathrm{Mn}, \mathrm{Zn}$ and $\mathrm{Fe}$ contents. K- fertigation increased leaf $\mathrm{N}, \mathrm{K}, \mathrm{Fe}$ and $\mathrm{Zn}$ and decreased $\mathrm{Ca}, \mathrm{Mg}$ and $\mathrm{Mn}$ contents.
\end{abstract}

Keywords: Date Palm, NP K Fertigation, Nitrogen and Potassium use efficiency

\section{Introduction}

Date palm is the most crop that widely grown in different distinct over all the world. It plays an important role in the economical and social life of the people and considered a symbol of life in desert in Egypt. It can grow and produce under different types of soil from light sandy to heavy clay soil. Date palm varieties are of three main types according to its fruit moisture content, i.e soft, semidry and dry. Seqae date palm cultivar is one of the best semi- dry. The influence of NPK fertigation on nitrogen and potassium use efficiency Clark,(1982); Hartley (2017); and Mohammad et al (2018) and mineral nutrition of date palm Kassem et al (1997); El- Shazly (2001); Abdel- Nasser\& Harhash (2001); Soliman\& Osman (2003); and; Shahin (2007); Osman (2010); Ezz et al., (2010); Al-Obeed et al. (2013) ; Ibrahim et al., (2013) and Salem \& Ali (2020). Potassium fertilization increased leaf N, K, Fe and Zn and decreased $\mathrm{Ca}, \mathrm{Mg}$ and $\mathrm{Mn}$ contents with the highest content by high rate. The percentage of leaf $\mathrm{N}$, $\mathrm{Ca}$ and $\mathrm{Mg}$ content was increased with nitrogen application and with increasing the nitrogen rate. (Ezz et al., 2010). The application of the larger amount of N, P and K increased the mineral content of leaves of Sewy date palm (Ibrahim et al., 2013). Leaf contents of N K Ca and Mn increased significantly by increasing the application of potassium, while $\mathrm{Fe}$ and $\mathrm{Zn}$ contents significantly decreased (Osman 2010). The potassium fertilizer increased the leaf $\mathrm{N}$ and $\mathrm{K}$ content of Zaghloul date palm (Kassem et al., 1997). Several investigators studied the effect of N, P, K fertigation on nitrogen use efficiency, 
potassium use efficiency and leaf mineral content of different date palm varieties. NPK are the three most needed nutrients by palms, which are commonly met through fertilizers. Therefore, this investigation was undertaken to study the effect of NPK fertigation on nitrogen use efficiency (NUE), potassium use efficiency (KUE) and mineral nutrition (N, P, K, Ca, Kg, Zn, Mn and Fe of Seqae leaves).

\section{Materials and Methods}

The present work carried out in Seqae date palm cultivar ( semi dry cv.) grown in a private orchard at El-Dakhala Oasis' El Wadi El jadid Governorate, Egypt during the three successive seasons to determine the NPK requirements. The palms tree were produced through the tissue culture technique. Palms were planted at space $8 \mathrm{X} 8 \mathrm{~m}$ apart. 36 female palm were selected as uniform as possible in growth, vigor , height, pollen source and age and were subject to the normal culture practices usually carried out for date palms. The trees were 11-year old at the beginning of this study. The experimental soil was classed as a clay loamy soil in texture. The drip irrigation with two lines single row and promising micro - flapper emitters was used five drippers per palm were giving a total discharge of about 60 liters/ hour to keep pressure losses. A factorial experiment with different rates of N,P,K were set up using a split- split plot design of three replicates. Three $\mathrm{N}$ - fertigation rates $(500,750,1000 \mathrm{~g}$ actual $\mathrm{N}$ / tree in the form of NH4NH3) were assigned to the main plots. The sub - plots were allotted to two $\mathrm{P}$ - fertigation rates $\left(400 \& 800 \mathrm{~g} \mathrm{P}_{2} \mathrm{O}_{5} / \mathrm{palm} /\right.$ year) in the form of $\mathrm{H} 3 \mathrm{PO} 4$. The sub- sub plots were devoted to K- fertigation using the rates $600 \& 1200 \mathrm{~g} \mathrm{~K}_{2} \mathrm{O} / \mathrm{palm} /$ year in the form of K2SO4. All the considered $\mathrm{N}$ and $\mathrm{K}$ rates divided into 80 unequal doses starting with February to least week of November while P rate was divided into 40 equal doses with February to November (Table1).

Table 1: Time and amount actual NPK fertigated around the year to the experimental seqae date palm.

\begin{tabular}{|c|c|c|c|c|c|c|c|c|c|c|}
\hline \multirow{2}{*}{$\begin{array}{l}\text { Treat. } \\
\text { Month }\end{array}$} & \multicolumn{3}{|c|}{ Actual N g./palm } & \multirow{2}{*}{$\begin{array}{l}\text { No. of } \\
\text { doses }\end{array}$} & \multicolumn{2}{|c|}{$\mathrm{P}_{2} \mathrm{O}_{5}$ g./palm } & \multirow{2}{*}{$\begin{array}{l}\text { No. of } \\
\text { doses }\end{array}$} & \multicolumn{2}{|c|}{$\mathrm{K}_{2} \mathrm{O}$ g./palm } & \multirow{2}{*}{$\begin{array}{l}\text { No. of } \\
\text { doses }\end{array}$} \\
\hline & 500 & 750 & 1000 & & 400 & 800 & & 600 & 1200 & \\
\hline Feb. & 66.6 & 100 & 133.3 & 8 & 40 & 80 & 4 & 40 & 80 & 8 \\
\hline Mar. & 33.33 & 50 & 66.66 & 8 & 40 & 80 & 4 & 80 & 160 & 8 \\
\hline Apr. & 66.6 & 100 & 133.3 & 8 & 40 & 80 & 4 & 40 & 80 & 8 \\
\hline May & 33.33 & 50 & 66.66 & 8 & 40 & 80 & 4 & 80 & 160 & 8 \\
\hline June & 66.6 & 100 & 133.3 & 8 & 40 & 80 & 4 & 40 & 80 & 8 \\
\hline July & 33.33 & 50 & 66.66 & 8 & 40 & 80 & 4 & 80 & 160 & 8 \\
\hline Aug. & 66.6 & 100 & 133.3 & 8 & 40 & 80 & 4 & 40 & 80 & 8 \\
\hline Sept. & 33.33 & 50 & 66.66 & 8 & 40 & 80 & 4 & 80 & 160 & 8 \\
\hline Oct. & 66.6 & 100 & 133.3 & 8 & 40 & 80 & 4 & 40 & 80 & 8 \\
\hline Nov. & 33.33 & 50 & 66.66 & 8 & 40 & 80 & 4 & 80 & 160 & 8 \\
\hline Total & 500 & 750 & 1000 & 80 & 400 & 800 & 40 & 600 & 1200 & 80 \\
\hline
\end{tabular}

The following parameters were used to evaluate the tested treatments.

\subsection{Nitrogen use efficiency (NUE):}

It calculated by the following equation

$$
N U E=\frac{\text { Yield (kgs./fed.) }}{\text { Nitrogen quantity ((kgs./fed.) }}
$$

Nitrogen use efficiency expressed as expressed as the amount of date palm fruit in kgs. , that could be produced from one $\mathrm{Kg}$ of nitrogen, according to Clark (1982) and Mohammed et al., (2018).

2.2. Potassium use efficiency (KUE):

It calculated by the following equation

$$
K U E=\frac{\text { Yield (kgs./fed.) }}{\text { Potassium quantity ((kgs./fed.) }}
$$

The method was described by Hartley (2017). Potassium use efficiency expressed as the amount of date palm fruit in kgs., that could be produced from one $\mathrm{Kg}$ of potassium $\left(\mathrm{K}_{2} \mathrm{O}\right)$. 


\subsection{Leaf mineral content:}

To determine leaf mineral content N,P, K, Ca, Mg, Zn and Fe leaf samples were taken during November and washed with tap water then with distilled water to remove the dust. After washing, they were dried in an electric oven at $70^{\circ}$ for 72 hours. The dried leaves were ground, digested and prepared by Wilde et al., (1985).

a. Total nitrogen was determined by using the micro jeldahl method as described by Pregl (1945)

b. Phosphorus was determined colorlmetrically according to A.O.A.C., (2005)

c. Potassium and calcium were determined using a flame- photometer according the method by Brown and Lilleland (1946)

d. Magnesium, manganese, zinc and iron were determined by using atonic spectrophotometer according to Brandifild and Spincer (1965)

\subsection{Statistical analysis}

The experiment was conducted as a randomized complete design with three replicates ( 1 replicate $=$ one palm) for each treatment ( 3 replicates X $3 \mathrm{~N}$ levels $\mathrm{X} 2 \mathrm{P}_{2} \mathrm{O}_{5}$ levels $\mathrm{X} 2 \mathrm{~K}_{2} \mathrm{O}$ levels ). Statistical analysis was carried out according to Snedecor and Cochran (1989). Mean separation was came out using the method of New least significant differences (New L.S.D) described by Waller \&Duncan, (1969).

\section{Results and Discussion}

3.1. Nitrogen use efficiency (NUE) as expressed as the amount of date palm fruit in $\mathrm{kg}$.that could be produced from one $\mathrm{Kg}$ of nitrogen. It's cleary that NUE showed a variable responses in respect to the studied treatment. Anhow NUE was improved in this concern with $500 \mathrm{~g} \mathrm{~N}$ than other used. Nitrogen quantity which the obtained values were 198,196,204 kg fruit/ one $\mathrm{Kg}$ nitrogen. Were 198,206,222 kg. fruit $/ \mathrm{kg}$. nitrogen $, 137.3,152,161.3 \mathrm{~kg}$.fruit $/ \mathrm{kg}$. nitrogen , 118,124,132 kg. fruit/ kg. nitrogen for T4(500 $\left.\mathrm{g} \mathrm{N}+800 \mathrm{P}_{2} \mathrm{O}_{5}+1200 \mathrm{~K}_{2} \mathrm{O}\right) \mathrm{T} 8\left(750 \mathrm{~g} \mathrm{~N}+800 \mathrm{~g} \mathrm{P}_{2} \mathrm{O}_{5}+1200 \mathrm{~g} \mathrm{~K}_{2} \mathrm{O}\right)$ and $\mathrm{T} 12\left(1000 \mathrm{~g} \mathrm{~N}+800 \mathrm{~g} \mathrm{P}_{2} \mathrm{O}_{5}+1200\right.$ $\mathrm{g} \mathrm{K}_{2} \mathrm{O}$ ) during three seasons respectively (Table 2) T12( $1000 \mathrm{~g} \mathrm{~N}+800 \mathrm{~g} \mathrm{P}_{2} \mathrm{O}_{5}+1200 \mathrm{~g} \mathrm{~K}_{2} \mathrm{O} /$ plant/year) produced NUK $(118,124,132 \mathrm{~kg}$ fruit/ one Kg. of nitrogen. These results agree with Clark (1982) and Mohammed et al (2018).

Table 2: Nitrogen and potassium use efficiency of Seqae date palm as affected by NPK fertigation

\begin{tabular}{ccccccc}
\hline \multirow{2}{*}{ Treatments(T) } & \multicolumn{3}{c}{ Nitrogen use efficiency } & \multicolumn{3}{c}{ Potassium use efficiency } \\
\cline { 2 - 6 } & $\mathbf{2 0 1 8}$ & $\mathbf{2 0 1 9}$ & $\mathbf{2 0 2 0}$ & $\mathbf{2 0 1 8}$ & $\mathbf{2 0 1 9}$ & $\mathbf{2 0 2 0}$ \\
\hline $\mathbf{T}_{\mathbf{1}}$ & 180 & 188 & 207.18 & 150 & 156.6 & 170 \\
$\mathbf{T}_{\mathbf{2}}$ & 188 & 196 & 214.0 & 78.33 & 81.66 & 89.16 \\
$\mathbf{T}_{\mathbf{3}}$ & 190 & 196 & 219.3 & 158.3 & 163.3 & 180.0 \\
$\mathbf{T}_{\mathbf{4}}$ & 198 & 206 & 222 & 82.5 & 85.8 & 90.83 \\
$\mathbf{T}_{\mathbf{5}}$ & 128 & 138.6 & 148 & 166.6 & 173.3 & 186.6 \\
$\mathbf{T}_{\mathbf{6}}$ & 133.3 & 144 & 157.3 & 83.3 & 90 & 98.3 \\
$\mathbf{T}_{\mathbf{7}}$ & 133.3 & 146.6 & 160 & 166.6 & 183.3 & 200 \\
$\mathbf{T}_{\mathbf{8}}$ & 137.3 & 152 & 161.3 & 88.3 & 95 & 100.83 \\
$\mathbf{T}_{\mathbf{9}}$ & 103 & 108 & 118 & 171.6 & 180 & 196.6 \\
$\mathbf{T}_{\mathbf{1 0}}$ & 110 & 116 & 122 & 91.6 & 96.6 & 101.6 \\
$\mathbf{T}_{\mathbf{1 1}}$ & 108 & 112 & 118 & 108 & 186.6 & 196.6 \\
$\mathbf{T}_{\mathbf{1 2}}$ & 118 & 124 & 132 & 98.3 & 103.3 & 110 \\
New L.S.D $\mathbf{a t} \mathbf{0 . 0 5}$ & 7.3 & 6.9 & 8.3 & 9.41 & 8.23 & 8.45 \\
\hline
\end{tabular}

$\mathbf{T}_{1} \quad$ 500g. N+400g. $\mathrm{P}_{2} \mathrm{O}_{5}+600 \mathrm{~g} . \mathrm{K}_{2} \mathrm{O} /$ palm

$\mathbf{T}_{2} \quad$ 500g.N +400 g. $\mathrm{P}_{2} \mathrm{O}_{5}+1200 \mathrm{~g} . \mathrm{K}_{2} \mathrm{O} / \mathrm{palm}$

$\mathrm{T}_{3} \quad$ 500g.N $+800 \mathrm{~g} . \mathrm{P}_{2} \mathrm{O}_{5}+600 \mathrm{~g} . \mathrm{K}_{2} \mathrm{O} / \mathrm{palm}$

$\mathrm{T}_{4} \quad$ 500g.N+800g. $\mathrm{P}_{2} \mathrm{O}_{5}+1200 \mathrm{~g} . \mathrm{K}_{2} \mathrm{O} / \mathrm{palm}$

T5 750g.N+400g. $\mathrm{P}_{2} \mathrm{O}_{5}+600 \mathrm{~g} . \mathrm{K}_{2} \mathrm{O} / \mathrm{palm}$

T6 750g.N+400g. $\mathrm{P}_{2} \mathrm{O}_{5}+1200$ g. $\mathrm{K}_{2} \mathrm{O} / \mathrm{palm}$
$\mathrm{T}_{7} \quad 750 \mathrm{~g} \cdot \mathrm{N}+800 \mathrm{~g} \cdot \mathrm{P}_{2} \mathrm{O}_{5}+600 \mathrm{~g} . \mathrm{K}_{2} \mathrm{O} /$ palm

T8 750g.N+800g. $\mathrm{P}_{2} \mathrm{O}_{5}+1200 \mathrm{~g} . \mathrm{K}_{2} \mathrm{O} / \mathrm{palm}$

T9 1000g.N+400g $\mathrm{P}_{2} \mathrm{O}_{5}+600 \mathrm{~g} . \mathrm{K}_{2} \mathrm{O} / \mathrm{palm}$

T10 1000g.N+400g $\mathrm{P}_{2} \mathrm{O}_{5}+1200 \mathrm{~g} . \mathrm{K}_{2} \mathrm{O} / \mathrm{palm}$

$\mathrm{T}_{11}$ 1000g.N+800g P $\mathrm{O}_{5}+600 \mathrm{~g} . \mathrm{K}_{2} \mathrm{O} / \mathrm{palm}$

T12 1000g.N+800gP ${ }_{2} \mathrm{O}_{5}+1200$ g. $\mathrm{K}_{2} \mathrm{O} / \mathrm{palm}$ 
3.2. Potassium use efficiency ( KUE) is expressed as the amount of Seqae date palm fruits in $\mathrm{kg}$, that could be produced from one $\mathrm{Kg}$ of potassium $\left(\mathrm{K}_{2} \mathrm{O}\right)$. KUE clearly affected by NPK fertigation, the results show that the highest value of KUE $(166.6,183.3,200 \mathrm{~kg}$ fruit Seqae date palm),(171.6,180,196.6kg fruit), $(108,186.6,196.6 \mathrm{Kg}$ fruit were obtained from $\mathrm{T} 7(750 \mathrm{~g} \mathrm{~N}+800 \mathrm{~g}$ $\left.\mathrm{P}_{2} \mathrm{O}_{5}+1200 \mathrm{~g} \mathrm{~K}_{2} \mathrm{O}\right)$, T9 (1000 g N+400 $\left.\mathrm{g} \mathrm{P}_{2} \mathrm{O}_{5}+600 \mathrm{~g} \mathrm{~K}_{2} \mathrm{O}\right)$ and T11(1000 g N+800 g P $\left.\mathrm{O}_{5}+600 \mathrm{~g} \mathrm{~K}_{2} \mathrm{O}\right)$ during three seasons respectively ( Table2), treatment 12 (1000 g N+800 g $\mathrm{P}_{2} \mathrm{O}_{5}+1200 \mathrm{~g} \mathrm{~K} 2 \mathrm{O} /$ plant/ year) produced $\mathrm{KUE}\left(98.3,103.3,110 \mathrm{Kg}\right.$ fruits/ one $\mathrm{Kg}$. $\left.\mathrm{K}_{2} \mathrm{O}\right)$.

\subsection{Mineral nutrition}

Macro and microelements were determined in the leaves of tested Seqae date palm at late November. This was carried out to declare the effect of different rates of N,P and $\mathrm{K}$ fertigation on their concentration in palm leaves. Date recorded in Tables (4,5 and 6) obviously reveal that concentration of elements in leaves of Seqae date palm showed different response in relation to different rates of $\mathrm{N}$ $\left(500,750,1000 \mathrm{~g} \mathrm{~N} / \mathrm{palm} /\right.$ year) $\mathrm{P}(400,800 \mathrm{~g} \mathrm{P} 2 \mathrm{O} 5 /$ palm/ year $)$ and $\mathrm{K}$ fertigation $\left(600,1200 \mathrm{~g} \mathrm{~K}_{2} \mathrm{O} /\right.$ palm/year) in the three seasons of investigation.

\subsection{Leaf nitrogen content $\%$}

The date in Table ( 3 ) show that $\mathrm{N}$ concentration in Leaf of treatment palms tended to increase by increasing rate of $\mathrm{N}$ - fertigation in the highest values of $\mathrm{N}$ concentration in this concern were noticed with $1000 \mathrm{~g} \mathrm{~N} /$ palm/year.

As for $\mathrm{N}$ concentration in leaves in response to $\mathrm{P}$ - fertigation $800 \mathrm{~g} \mathrm{P}_{2} \mathrm{O}_{5}$ treatment gave the highest amount against $400 \mathrm{~g} \mathrm{P}_{2} \mathrm{O}_{5} / \mathrm{palm} /$ year Depressing of $\mathrm{K}$ - fertigation on nitrogen concentration in Seqae leaves in the three examined seasons instance, nitrogen concentration in leaves were decreased by increasing $\mathrm{K}$ - fertigation rates. The highest values for $\mathrm{N}$ concentration results from $1000 \mathrm{~g} \mathrm{~N}+800 \mathrm{~g}$ $\mathrm{P}_{2} \mathrm{O}_{5}+600 \mathrm{~g} \mathrm{~K}_{2} \mathrm{O} / \mathrm{palm} /$ year. The available literature were generally in agreement with obtained results Kassem et al (1997); El- Shazly(2001); Abdel- Nasser\& Harhash (2001); El-Sayed (2002) Soliman\& Osman (2003); and; Shahin (2007); Osman (2010); Ezz et al., (2010); Al-Obeed et al (2013) ; Ibrahim et al., (2013) and Salem \& Ali (2020).

Table 3: Leaf N, P, K content (\%) of Seqae date palm as affected by NPK fertigation during three seasons

\begin{tabular}{clllllllll}
\hline \multirow{2}{*}{ Treatments(T) } & \multicolumn{3}{l}{ Leaf N content (\%) } & \multicolumn{3}{c}{ Leaf P content (\%) } & \multicolumn{3}{c}{ Leaf K content (\%) } \\
\cline { 2 - 10 } & $\mathbf{2 0 1 8}$ & $\mathbf{2 0 1 9}$ & $\mathbf{2 0 2 0}$ & $\mathbf{2 0 1 8}$ & $\mathbf{2 0 1 9}$ & $\mathbf{2 0 2 0}$ & $\mathbf{2 0 1 8}$ & $\mathbf{2 0 1 9}$ & $\mathbf{2 0 2 0}$ \\
\hline $\mathbf{T}_{\mathbf{1}}$ & 1.42 & 1.31 & 1.49 & 0.211 & 0.272 & 0.320 & 1.51 & 1.62 & 1.54 \\
$\mathbf{T}_{\mathbf{2}}$ & 1.33 & 1.22 & 1.44 & 0.240 & 0.291 & 0.341 & 1.56 & 1.64 & 1.57 \\
$\mathbf{T}_{\mathbf{3}}$ & 1.60 & 1.51 & 1.64 & 0.261 & 0.351 & 0.374 & 1.49 & 1.57 & 1.50 \\
$\mathbf{T}_{\mathbf{4}}$ & 1.52 & 1.43 & 1.56 & 0.281 & 0.382 & 0.391 & 1.50 & 1.59 & 1.53 \\
$\mathbf{T}_{\mathbf{5}}$ & 1.64 & 1.55 & 1.70 & 0.182 & 0.261 & 0.274 & 1.40 & 1.50 & 1.48 \\
$\mathbf{T}_{\mathbf{6}}$ & 1.62 & 1.44 & 1.63 & 0.194 & 0.282 & 0.293 & 1.42 & 1.53 & 1.51 \\
$\mathbf{T}_{\mathbf{7}}$ & 1.77 & 1.64 & 1.78 & 0.221 & 0.321 & 0.331 & 1.34 & 1.46 & 1.44 \\
$\mathbf{T}_{\mathbf{8}}$ & 1.70 & 1.60 & 1.75 & 0.24 & 0.341 & 0.362 & 1.39 & 1.49 & 1.48 \\
$\mathbf{T}_{\mathbf{9}}$ & 1.72 & 1.75 & 1.83 & 0.11 & 0.21 & 0.231 & 1.30 & 1.50 & 1.44 \\
$\mathbf{T}_{\mathbf{1 0}}$ & 1.69 & 1.70 & 1.80 & 0.142 & 0.241 & 0.26 & 1.34 & 1.44 & 1.49 \\
$\mathbf{T}_{\mathbf{1 1}}$ & 1.91 & 1.80 & 1.87 & 0.183 & 0.28 & 0.331 & 1.32 & 1.42 & 1.43 \\
$\mathbf{T}_{\mathbf{1 2}}$ & 1.80 & 1.75 & 1.81 & 0.204 & 0.301 & 0.354 & 1.38 & 1.44 & 1.44 \\
New L.S.D at $\mathbf{0 . 0 5}$ & 0.09 & 0.08 & 0.09 & 0.014 & 0.013 & 0.016 & 0.12 & 0.13 & 0.14 \\
\hline
\end{tabular}

$\mathbf{T}_{1} \quad 500 \mathrm{~g} . \mathrm{N}+400 \mathrm{~g} . \mathrm{P}_{2} \mathrm{O}_{5}+600 \mathrm{~g} . \mathrm{K}_{2} \mathrm{O} / \mathrm{palm}$

$\mathbf{T}_{2} \quad$ 500g. N+400g. $\mathrm{P}_{2} \mathrm{O}_{5}+1200$ g. $\mathrm{K}_{2} \mathrm{O} /$ palm

$\mathbf{T}_{3} \quad$ 500g.N+800g. $\mathrm{P}_{2} \mathrm{O}_{5}+600 \mathrm{~g} . \mathrm{K}_{2} \mathrm{O} / \mathrm{palm}$

$\mathbf{T}_{4} \quad$ 500g.N+800g. $\mathrm{P}_{2} \mathrm{O}_{5}+1200 \mathrm{~g} . \mathrm{K}_{2} \mathrm{O} / \mathrm{palm}$

$\mathrm{T}_{5} \quad 750 \mathrm{~g} \cdot \mathrm{N}+400 \mathrm{~g} \cdot \mathrm{P}_{2} \mathrm{O}_{5}+600 \mathrm{~g} . \mathrm{K}_{2} \mathrm{O} / \mathrm{palm}$

$\mathbf{T}_{6} \quad 750$ g.N+400g. $\mathrm{P}_{2} \mathrm{O}_{5}+1200$ g. $\mathrm{K}_{2} \mathrm{O} /$ palm
$\mathbf{T}_{7} \quad 750 \mathrm{~g} \cdot \mathrm{N}+800 \mathrm{~g} \cdot \mathrm{P}_{2} \mathrm{O}_{5}+600 \mathrm{~g} . \mathrm{K}_{2} \mathrm{O} / \mathrm{palm}$

$\mathbf{T}_{\mathbf{8}} \quad 750 \mathrm{~g} . \mathrm{N}+800 \mathrm{~g} . \mathrm{P}_{2} \mathrm{O}_{5}+1200 \mathrm{~g} . \mathrm{K}_{2} \mathrm{O} / \mathrm{palm}$

T9 1000g. N+400g $\mathrm{P}_{2} \mathrm{O}_{5}+600 \mathrm{~g} . \mathrm{K}_{2} \mathrm{O} / \mathrm{palm}$

$\mathbf{T}_{10} \quad 1000 \mathrm{~g} . \mathrm{N}+400 \mathrm{~g} \mathrm{P}_{2} \mathrm{O}_{5}+1200 \mathrm{~g} . \mathrm{K}_{2} \mathrm{O} / \mathrm{palm}$

$\mathrm{T}_{11}$ 1000g.N+800g P $\mathrm{O}_{5}+600 \mathrm{~g} . \mathrm{K}_{2} \mathrm{O} / \mathrm{palm}$

T12 1000g.N+800gP $\mathrm{O}_{5}+1200$ g. $\mathrm{K}_{2} \mathrm{O} /$ palm

\subsection{Leaf phosphorus content $\%$}

The obtained data show clear reductions in leaf $\mathrm{P}$ concentration as $\mathrm{N} /$ palm/year gave the highest concentration. Leaf P contact increased by increasing P- fertigation rates. In agreement with the 
obtained herein results El-Sayed (2002) Soliman\& Osman (2003); and; Shahin (2007); Osman (2010); Ezz et al., (2010); Al-Obeed et al (2013) ; Ibrahim et al., (2013) and Salem \& Ali (2020). Regarding leaf $\mathrm{P}$ contact in response to $\mathrm{K}$ - fertigation rate $1200 \mathrm{~g} \mathrm{~K}_{2} \mathrm{O} / \mathrm{palm} /$ year treatment gave the highest concentration as compared with other treatments. It was clear that the treatment $(1000 \mathrm{~g} \mathrm{~N}+800 \mathrm{~g}$ $\mathrm{P}_{2} \mathrm{O}_{5}+1200 \mathrm{~g} \mathrm{~K}_{2} \mathrm{O}$ ) was the most effective in Leaf $\mathrm{P}$ content.

\subsection{Leaf potassium content $\%$}

The obtained results show that leaf $\mathrm{K}$ content tended to decrease with increasing rate of $\mathrm{N}$ fertigation. As for K- concentration in Seqae leaves in response to P-fertigation rate, treatment $400 \mathrm{~g}$ $\mathrm{P}_{2} \mathrm{O}_{5} / \mathrm{palm} /$ year gave the highest values. $\mathrm{K}$ concentration in leaves tended to increase as $\mathrm{K}$-fertigation rates were interested. Leaf $\mathrm{K}$ content was highest with $500 \mathrm{~g} \mathrm{~N}+400 \mathrm{~g} \mathrm{P}_{2} \mathrm{O}_{5}+1200 \mathrm{~g} \mathrm{~K} 2 \mathrm{O} / \mathrm{palm} /$ year. In agreement with the obtained herein results El-Sayed (2002) Soliman\& Osman (2003); and; Shahin (2007); Osman (2010); Ezz et al., (2010); Al-Obeed et al (2013) ; Ibrahim et al., (2013) and Salem \& Ali (2020).

\subsection{Leaf calcium and magnesium content $\%$}

Leaf $\mathrm{Ca}$ and $\mathrm{Mg}$ content in Table(4) significantly varied due to NPK fertigation. In this concern, an inverse relationship between $\mathrm{N}$-fertigation rate and $\mathrm{Ca}, \mathrm{Mg}$ concentration in the Seqae leaves was detected. Thus leaves of low rate of nitrogen treatment recorded higher $\mathrm{Ca} \& \mathrm{Mg}$ content in comparison with leaves of high $\mathrm{N}$ - rate treatment. $400 \mathrm{~g} \mathrm{P}_{2} \mathrm{O}_{5} / \mathrm{palm} /$ year treatment increased $\mathrm{Ca} \& \mathrm{Mg}$ content in the leaves to the maximum. Leaf $\mathrm{Ca} \& \mathrm{Mg}$ content tended to increase with decreasing $\mathrm{K}$ - fertigation rate.

Table 4: Leaf $\mathrm{Ca}$ and $\mathrm{Mg}$ content (\%) of Seqae date palm as affected by NPK fertigation during three seasons.

\begin{tabular}{ccccccc}
\hline \multirow{2}{*}{ Treatments(T) } & \multicolumn{3}{c}{ Leaf Ca content (\%) } & \multicolumn{2}{c}{ Leaf Mg content (\%) } \\
\cline { 2 - 7 } & $\mathbf{2 0 1 8}$ & $\mathbf{2 0 1 9}$ & $\mathbf{2 0 2 0}$ & $\mathbf{2 0 1 8}$ & $\mathbf{2 0 1 9}$ & $\mathbf{2 0 2 0}$ \\
\hline $\mathbf{T}_{\mathbf{1}}$ & 0.993 & 0.821 & 1.09 & 0.410 & 0.234 & 0.364 \\
$\mathbf{T}_{\mathbf{2}}$ & 0.924 & 0.770 & 0.91 & 0.400 & 0.211 & 0.350 \\
$\mathbf{T}_{\mathbf{3}}$ & 1.06 & 0.924 & 1.18 & 0.440 & 0.244 & 0.82 \\
$\mathbf{T}_{\mathbf{4}}$ & 1.03 & 0.875 & 1.11 & 0.431 & 0.238 & 0.374 \\
$\mathbf{T}_{\mathbf{5}}$ & 0.721 & 0.701 & 0.930 & 0.371 & 0.228 & 0.342 \\
$\mathbf{T}_{\mathbf{6}}$ & 0.703 & 0.681 & 0.840 & 0.362 & 0.223 & 0.334 \\
$\mathbf{T}_{\mathbf{7}}$ & 0.812 & 0.744 & 0.960 & 0.370 & 0.231 & 0.351 \\
$\mathbf{T}_{\mathbf{8}}$ & 0.782 & 0.721 & 0.880 & 0.360 & 0.228 & 0.340 \\
$\mathbf{T}_{\mathbf{9}}$ & 0.651 & 0.603 & 0.840 & 0.354 & 0.212 & 0.327 \\
$\mathbf{T}_{\mathbf{1 0}}$ & 0.604 & 0.591 & 0.731 & 0.341 & 0.200 & 0.314 \\
$\mathbf{T}_{\mathbf{1 1}}$ & 0.715 & 0.731 & 0.871 & 0.381 & 0.220 & 0.331 \\
$\mathbf{T}_{\mathbf{1 2}}$ & 0.686 & 0.703 & 0.820 & 0.377 & 0.231 & 0.328 \\
New L.S.D at $\mathbf{0 . 0 5}$ & 0.094 & 0.088 & 0.061 & 0.71 & 0.042 & 0.06 \\
\hline
\end{tabular}

$\mathbf{T}_{1} \quad$ 500g. N+400g. $\mathrm{P}_{2} \mathrm{O}_{5}+600$ g. $\mathrm{K}_{2} \mathrm{O} / \mathrm{palm}$

$\mathbf{T}_{2} \quad$ 500g. N+400g. $\mathrm{P}_{2} \mathrm{O}_{5}+1200$ g. $\mathrm{K}_{2} \mathrm{O} /$ palm

$\mathbf{T}_{3} \quad$ 500g.N+800g. $\mathrm{P}_{2} \mathrm{O}_{5}+600 \mathrm{~g} . \mathrm{K}_{2} \mathrm{O} / \mathrm{palm}$

$\mathbf{T}_{4} \quad$ 500g. +800 g. $\mathrm{P}_{2} \mathrm{O}_{5}+1200 \mathrm{~g} . \mathrm{K}_{2} \mathrm{O} / \mathrm{palm}$

$\mathrm{T}_{5} \quad 750 \mathrm{~g} . \mathrm{N}+400 \mathrm{~g} . \mathrm{P}_{2} \mathrm{O}_{5}+600 \mathrm{~g} . \mathrm{K}_{2} \mathrm{O} / \mathrm{palm}$

$\mathrm{T}_{6} \quad 750$ g. $\mathrm{N}+400$ g. $\mathrm{P}_{2} \mathrm{O}_{5}+1200$ g. $\mathrm{K}_{2} \mathrm{O} /$ palm
$\mathbf{T}_{7} \quad 750 \mathrm{~g} \cdot \mathrm{N}+800 \mathrm{~g} \cdot \mathrm{P}_{2} \mathrm{O}_{5}+600 \mathrm{~g} . \mathrm{K}_{2} \mathrm{O} / \mathrm{palm}$

$\mathrm{T}_{8} \quad 750$ g.N +800 g. $\mathrm{P}_{2} \mathrm{O}_{5}+1200$ g. $\mathrm{K}_{2} \mathrm{O} / \mathrm{palm}$

T9 1000g. N+400g $\mathrm{P}_{2} \mathrm{O}_{5}+600 \mathrm{~g} . \mathrm{K}_{2} \mathrm{O} / \mathrm{palm}$

$\mathbf{T}_{10}$ 1000g. $\mathrm{N}+400 \mathrm{~g} \mathrm{P}_{2} \mathrm{O}_{5}+1200 \mathrm{~g} . \mathrm{K}_{2} \mathrm{O} / \mathrm{palm}$

$\mathbf{T}_{11}$ 1000g.N+800g $\mathrm{P}_{2} \mathrm{O}_{5}+600 \mathrm{~g} . \mathrm{K}_{2} \mathrm{O} / \mathrm{palm}$

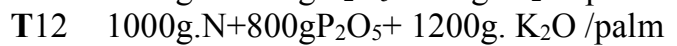

\subsection{Leaf Mn content (ppm)}

Leaf $\mathrm{Mn}$ concentration significantly varied due to $\mathrm{N}$ and $\mathrm{K}$ fertigation leaf $\mathrm{Mn}$ content tended to increase with increasing $\mathrm{N}$, K-fertigation. Leaf Mn concentration in significant varied due to Pfertigation. Analogical results in this respect were reported by many investigators.

\subsection{Leaf $Z n$ content (ppm)}

Leaf $\mathrm{Zn}$ content insignificantly varied due to $\mathrm{N}$ and $\mathrm{P}$ - fertigation. As for $\mathrm{Zn}$ content in response to $\mathrm{K}$-fertigation rate $1200 \mathrm{~g} \mathrm{~K}_{2} \mathrm{O} /$ palm/year gave the highest values. In agreement with obtained herein 
results; Osman (2010); Ezz et al., (2010); Al-Obeed et al (2013) ; Ibrahim et al., (2013) and Salem \& Ali (2020).

\subsection{Leaf Fe content (ppm)}

The date in Table (5) show that Fe concentration in Leaf tended to increase by increasing rate of $\mathrm{N}$ fertigation in the three tested seasons. An inverse relation between P \& K- fertigation rate and Fe concentration in the leaves was detected. Thus, l leaves of low rate of P\& K- fertigation treatment recorded higher $\mathrm{Fe}$ content in comparison with leaves high $\mathrm{P} \& \mathrm{~K}$ rate treatment. The treatments which gave the uppermost significantly values in the three seasons were $1000 \mathrm{~g} \mathrm{~N}+400 \mathrm{~g} \mathrm{P}_{2} \mathrm{O}_{5}+600 \mathrm{~g} \mathrm{~K}_{2} \mathrm{O}$ (treatment 9). Analogical results were obtained by; Osman (2010); Ezz et al., (2010); Al-Obeed et al (2013) ; Ibrahim et al., (2013) and Salem \& Ali (2020).

Table 5: leaf Mn, $\mathrm{Zn}$ and Fe content (ppm) of Seqae date palm fertigation during three seasons.

\begin{tabular}{cccccccccc}
\hline & \multicolumn{3}{c}{$\begin{array}{c}\text { Leaf Mn content } \\
\text { Treatments(T) }\end{array}$} & \multicolumn{2}{c}{$\begin{array}{c}\text { Leaf Zn content } \\
\text { (ppm) }\end{array}$} & \multicolumn{2}{c}{$\begin{array}{c}\text { Leaf Fe content } \\
\text { (ppm) }\end{array}$} \\
\cline { 2 - 10 } & $\mathbf{2 0 1 8}$ & $\mathbf{2 0 1 9}$ & $\mathbf{2 0 2 0}$ & $\mathbf{2 0 1 8}$ & $\mathbf{2 0 1 9}$ & $\mathbf{2 0 2 0}$ & $\mathbf{2 0 1 8}$ & $\mathbf{2 0 1 9}$ & $\mathbf{2 0 2 0}$ \\
\hline $\mathbf{T}_{\mathbf{1}}$ & 74 & 93 & 70 & 22 & 20 & 14 & 110 & 121 & 134 \\
$\mathbf{T}_{\mathbf{2}}$ & 79 & 66 & 74 & 25 & 24 & 16 & 104 & 105 & 122 \\
$\mathbf{T}_{\mathbf{3}}$ & 73 & 62 & 71 & 22 & 20 & 14 & 108 & 118 & 130 \\
$\mathbf{T}_{\mathbf{4}}$ & 78 & 67 & 75 & 26 & 22 & 17 & 106 & 112 & 120 \\
$\mathbf{T}_{\mathbf{5}}$ & 88 & 72 & 77 & 22 & 20 & 13 & 116 & 127 & 144 \\
$\mathbf{T}_{\mathbf{6}}$ & 91 & 74 & 79 & 24 & 22 & 14 & 110 & 117 & 137 \\
$\mathbf{T}_{\mathbf{7}}$ & 89 & 73 & 77 & 20 & 20 & 13 & 115 & 124 & 144 \\
$\mathbf{T}_{\mathbf{8}}$ & 90 & 74 & 80 & 24 & 24 & 16 & 110 & 115 & 145 \\
$\mathbf{T}_{\mathbf{9}}$ & 96 & 81 & 83 & 21 & 19 & 14 & 125 & 122 & 144 \\
$\mathbf{T}_{\mathbf{1 0}}$ & 97 & 85 & 85 & 25 & 24 & 16 & 118 & 112 & 135 \\
$\mathbf{T}_{\mathbf{1 1}}$ & 94 & 80 & 82 & 24 & 20 & 14 & 122 & 120 & 140 \\
$\mathbf{T}_{\mathbf{1 2}}$ & 97 & 86 & 86 & 27 & 25 & 17 & 117 & 114 & 133 \\
New L.S.D at $\mathbf{0 . 0 5}$ & 3.31 & 4.2 & 4.35 & 2.21 & 3.14 & 2.37 & 4.5 & 4.6 & 4.92 \\
\hline
\end{tabular}

$\mathbf{T}_{1} \quad$ 500g. N+400g. $\mathrm{P}_{2} \mathrm{O}_{5}+600$ g. $\mathrm{K}_{2} \mathrm{O} / \mathrm{palm}$

$\mathbf{T}_{2} \quad$ 500g. N+400g. $\mathrm{P}_{2} \mathrm{O}_{5}+1200 \mathrm{~g} . \mathrm{K}_{2} \mathrm{O} / \mathrm{palm}$

$\mathbf{T}_{3} \quad$ 500g.N+800g. $\mathrm{P}_{2} \mathrm{O}_{5}+600 \mathrm{~g} . \mathrm{K}_{2} \mathrm{O} / \mathrm{palm}$

$\mathbf{T}_{4}$ 500g.N+800g. $\mathrm{P}_{2} \mathrm{O}_{5}+1200$ g. $\mathrm{K}_{2} \mathrm{O} /$ palm

$\mathbf{T}_{5} \quad 750 \mathrm{~g} . \mathrm{N}+400 \mathrm{~g} . \mathrm{P}_{2} \mathrm{O}_{5}+600 \mathrm{~g} . \mathrm{K}_{2} \mathrm{O} / \mathrm{palm}$

T6 750g.N+400g.P $\mathrm{P}_{5}+1200$ g. $\mathrm{K}_{2} \mathrm{O} /$ palm
$\mathbf{T}_{7} \quad 750 \mathrm{~g} . \mathrm{N}+800 \mathrm{~g} . \mathrm{P}_{2} \mathrm{O}_{5}+600 \mathrm{~g} . \mathrm{K}_{2} \mathrm{O} / \mathrm{palm}$

T8 750g.N+800g. $\mathrm{P}_{2} \mathrm{O}_{5}+1200 \mathrm{~g} . \mathrm{K}_{2} \mathrm{O} / \mathrm{palm}$

T9 $1000 \mathrm{~g} . \mathrm{N}+400 \mathrm{~g} \mathrm{P}_{2} \mathrm{O}_{5}+600 \mathrm{~g} . \mathrm{K}_{2} \mathrm{O} / \mathrm{palm}$

$\mathbf{T}_{10} \quad 1000 \mathrm{~g} . \mathrm{N}+400 \mathrm{~g} \mathrm{P}_{2} \mathrm{O}_{5}+1200 \mathrm{~g} . \mathrm{K}_{2} \mathrm{O} / \mathrm{palm}$

$\mathbf{T}_{11}$ 1000g.N $+800 \mathrm{~g} \mathrm{P}_{2} \mathrm{O}_{5}+600 \mathrm{~g} . \mathrm{K}_{2} \mathrm{O} / \mathrm{palm}$

T12 1000g.N+800gP $\mathrm{gO}_{5}+1200 \mathrm{~g} . \mathrm{K}_{2} \mathrm{O} / \mathrm{palm}$

\section{References}

Abdel-Nasser, G. and M.M. Harhash, 2001. Studies on some plant growing Media for olive cultivation in sandy soils under Siwa Oasis Conditions. J. of the Advances in Agric. Res. Univ. of Alex. Fac. of Agric. Saba Bacha, 6(2):487-510.

Al-Obeed, R.S., H.A. Kassem and M.A. Ahmed, 2013. Effect of levels and methods of potassium and phosphorus fertilization on yield, fruit quality and chemical composition of "Khalas" date palm cultivar. Life Science Journal, 10(4): 1111-1118.

A.O.A.C. 2005. Association of Official Analytical Chemists, Official Methods of Analysis, 26th edition. Washington D.C., USA.

Brandfield, E.G. and D. Spineer, 1965. Leaf analysis as a guide to nutrition of plant crops, IV Determination of manganese Zinc and Copper by Atomic absorption spectroscopy. J. Sci. Food Agric., 16:33-38.

Brown. J. D. and O. Lilleland, 1946. Rapid determination of sodium in plant material and soil extracts by flame photometry. Proc. Amer. Soc. Hort. Sci., 73:813

Clark, R.B., 1982. Plant response to mineral element toxicity and deficiency pp 71-142 in Breeding plant for less factorable environment M.N. Christiansen and C.F. Lewis Eds. John Wiley and Sons, Inc.

El-Sayed, E.H., 2002. Effect of NPK fertigation on mineral nutrition of Fig trees in newly reclaimed soil. Egypt. J. Appl. Sci., 17 (10): 742-758. 
El-Shazly, S.M. and G. Abdel-Nasser, 2001. Response of picual olive trees to potassium and boron fertigation 2. Fruit set, yield, oil content, water use-efficiency and fruit quality. J. Adv. Agric. Res., 6(3):651-669.

Ezz T.M., H.A. Kassem and H.A. Marzouk, 2010. Response of date palm trees to different nitrogen and potassium application rates. Acta Hortic., 882.84.

Hartley, T.N., 2017. Increasing the potassium use efficiency of crops. PhD thesis, University of York.

Ibrahim, M.M., El-Beshbeshy, R.T., Kamh, A.R. and A.I. AbourAmer, 2013. Effect of NPK and biofertilizer on date palm trees grown in Siwa Oasis, Egypt. Soil, 29 (3): 315-321.

Kassem, H.A., M.B. El-Sabrout and M.M. Attia, 1997. Effect of nitrogen and potassium fertilization on yield, fruit quality and leaf mineral content in some Egyptian soft date cultivars. Alex. J. Agric. Res., 42 (1):137-157.

Mohammad, I.A., A.A. Gad, A. Bardisi, and A.A. Mohsen, 2018. Effect of planting Dates and nitrogen sources on dry weight, yield and nitrogen use efficiency of Garlic plant grown under south sinai Governorate conditions. Zagazig J. Agric.Res.Col.,45 No.45 (6A)

Osman, S.M., 2010. Effect of potassium fertilization on yield, leaf mineral content and fruit quality of Bartamoda date palm propagated by tissue culture technique under Aswan conditions', J. Applied Sci. Res., 6(2): 184-190.

Pregl, F., 1945. Quantitive organic Micro- Analysis 4th.ed. J\&M. Churchill, Ltd., London

Salem, E.H. and H.A.M. Ali., 2020. Effect of slow release fertilizers on growth and fruiting of Khalas date palm. International Journal of Agricultural Sciences, 2(2): 30-44.

Shahin, M., 2007. Effect of potassium fertilization on growth, leaves nutrient content, and yield of "Khalas" date palm in Al-Hassa Oasis (K.S.A.). The Fourth Symposium on date palm in Saudi Arabia, Date Palm Research Center; King Faisal University Al Hassa, 5-8 May.

Soliman, S.S. and S.M., Osman, 2003. Effect of nitrogen and potassium fertilization on yield, fruit quality and some nutrients content of Samany date palm. Ann Agric. Sci., Cairo 48: 283-296

Waller, R.A. and D.B. Duncan, 1969. A bayes rule for the symmetric multiple comparisons problems. Amer. State. Assoc. J., 64:1484-1503

Wilde, S.A., R.B. Corey, J.G. Lyer and G.K. Voigt, 1985. Soil and plant Analysis for Tree culture 3rd Ed. Pp. 89-100. Oxford IBLT publishing Co., New Delhi. 